\title{
Rising from the Floor in Older Adults
}

\author{
Neil B. Alexander, MD, Jessica Ulbrich, BS, Aarti Raheja, and Dwight Channer, MS
}

OBJECTIVE: The primary goal was to determine the ability of older adults to rise from the floor. A secondary goal was to explore how rise ability might differ based on initial body positions and with or without the use of an assistive device.

DESIGN: Cross-sectional analysis of young, healthy older, and congregate housing older adults.

SETTING: University-based laboratory and congregate housing facility.

PARTICIPANTS: Young adult controls $(12$ men and 12 women, mean age 23 years), healthy older adults ( 12 men and 12 women, mean age 73 years), and congregate housing older adults ( 32 women and 6 men, mean age 80 years). The healthy older adult women ( $\mathrm{n}=12$, mean age 75 years) and a subset of the congregate housing women $(n=27$, mean age 81 years) were identified for further analyses.

INTERVENTION: Videotaping and timing of rising from the floor from controlled initial body positions (supine, on side, prone, all fours, and sitting) and with or without the use of a furniture support.

MAIN OUTCOME MEASURES: Whether subjects were successful in rising, and if they were, the time taken to rise. Subjects also rated their perceived difficulty of the task as compared to the reference task, rising from a supine position.

RESULTS: Older adults have more difficulty rising from the floor than younger adults. The healthy old took twice as long as the young to rise, whereas the congregate old took two to three times as long as the healthy old to rise. Although all young and healthy old rose from every position, a subset of the congregate housing residents was unable to rise from any position, $24 \%$ when attempting to rise without a support and $13 \%$ when attempting to rise with a support. Congregate old were most likely to be successful when rising from a side-lying position while using the furniture for support. The more able congregate old, as well as the young and healthy old, rose more quickly and admitted to the least difficulty when rising from the all fours position.

CONCLUSIONS: The inability to rise from the floor is relatively common in congregate housing older adults. Based

\footnotetext{
From the Division of Geriatric Medicine, Department of Internal Medicine, University of Michigan; and the Geriatric Research, Education and Clinical Center, Department of Veterans Affairs Medical Center, Ann Arbor Michigan.

This material is based on work supported by National Institute on Aging Grants AG00519 and AG10542 and the Rehabilitation Research and Development Service, Department of Veterans Affairs.

Presented, in part at the 1995 American Geriatrics Society Annual Meeting. Address correspondence and reprint requests to Neil Alexander MD, Geriatrics Center, 300 North Ingalls Building, Ann Arbor, MI 48109.
}

on the differences between groups in time to complete the rise, determining the differences in rise strategies, and the underlying biomechanical requirements of rising from different positions with or without a support would appear to be useful. These data may serve as the foundation for future interventions to improve the ability to rise from the floor. J Am Geriatr Soc 45:564-569, 1997.

\section{BACKGROUND}

Difficulty in rising from the floor after a fall is common in older adults, is associated with substantial morbidity, and tends to be underappreciated. ${ }^{1}$ Only $49 \%$ of communitydwelling fallers are able to get up after a fall without assistance, and most of the falls associated with the inability to get up without help (85\%) are not associated with serious injury. ${ }^{1}$ Thus, the inability to get up after a fall is common and not simply a consequence of a fall-related injury. Up to $20 \%$ of fallers remain on the floor for 1 hour or more, ${ }^{2-5}$ and dehydration, pressure sores, muscle injury, and renal failure may be associated with these "long-lie" falls. ${ }^{4,6}$ Fear of falling appears to be increased in previous fallers, particularly those with a history of difficulty rising alone after a fall. ${ }^{7}$ Despite the high risk of difficulty in rising from the floor after a fall, few therapists teach older adults how to rise from the floor. ${ }^{8}$

Some researchers have analyzed the motions used to rise from a supine position on the floor to a standing position, ${ }^{9-11}$ finding that movement patterns differ somewhat as age increases from 30 to $59^{12}$ and when comparing sedentary to physically active adults aged 30 to $39 .{ }^{13}$ Few studies have included healthy or frail older adults. The purpose of the present study was to determine the ability of older adults to rise from the floor. A secondary goal was to explore how rise ability might differ according to initial body positions and with or without the use of an assistive device. We hypothesized that, with increased age and physical impairment, success in rising would decrease, and in successful rises, the rise speed would decrease. We also hypothesized that, starting from a hands and knees position ("all fours," see below for full description) and using a low lying piece of furniture for support would both increase floor rise success, and when subjects were successful, decrease the time taken to rise. These data can serve as the basis for understanding how rising from the floor changes with age and with underlying disease-associated impairments and for developing training methods to improve floor rise ability.

\section{METHODS}

\section{Subjects}

We recruited subjects from three locations: young adult controls (group YC, $n=24,12$ men and 12 women, mean 
age 23 years, range 19 to 30 years); community-dwelling healthy older adults (group $\mathrm{HO}, \mathrm{n}=24,12$ men and 12 women, mean age 73 years, range 66 to 87 years), and congregate housing-dwelling older adults (group $\mathrm{CO}, \mathrm{n}=38$, 32 women and 6 men, mean age 80 years, range 63 to 94). HO female subjects ( $\mathrm{HOF}, \mathrm{n}=12$, mean age 75 years, range 66-87 years), and a subset of the CO female subjects (COF, $\mathrm{n}=27$, mean age 81 years, range 68 to 94 years) were identified for rise time analyses as described below. COF were significantly older than HOF ( $P<.01$ by independent $t$ test). Body mass index did not differ significantly between the $Y C$ (mean 22) and HO (mean 23) or between the HOF (mean 23) and $\mathrm{COF}$ (mean 26).

A description of $\mathrm{YC}$ and $\mathrm{HO}$ recruitment and group characteristics follows. YC were recruited from a group of healthy undergraduate and graduate students. HO were recruited from among those who had previously participated or had indicated a willingness to participate in university research projects. Both $\mathrm{YC}$ and $\mathrm{HO}$ denied any significant musculoskeletal, otological, or neurological abnormality. Upon further screening history and physical examination by a gerontologic nurse clinician, a number of subtle abnormalities were reported (see Table 1). Four HO subjects (17\%) noted rare, occasional lower extremity or back pain that was not an active problem. Ten subjects $(42 \%)$ exhibited decreased or asymmetric lower extremity reflexes primarily at the Achilles tendon, and three subjects $(13 \%)$ had decreased vibratory sense at the ankle. Despite these subtle abnormalities, nearly all $(83 \%)$ of the subjects were involved three times a week in an exercise routine that included walking, biking, rowing, swimming, tennis, and/or yardwork.

The CO were more impaired (see Table 1). Although eight subjects $(21 \%)$ noted only occasional back or lower extremity pain, $13(34 \%)$ had daily back or lower extremity pain that interfered with their activities of daily living (ADLs). Ten subjects (26\%) had fallen in the past year and 18 $(47 \%)$ complained of poor balance that interfered with their ADLs. Weakness (as defined by less than $5 / 5$ strength on examination) was found in the upper extremity in $10(26 \%)$ and in the lower extremity in $14(37 \%)$. Nine subjects $(24 \%)$ had a positive Romberg test. Eight subjects $(21 \%)$ used an assistive device for ambulation, and four (11\%) required assistive device or furniture support for transferring from a bed to a chair. Decreased lower extremity reflexes were noted in nearly all subjects $(92 \%)$, decreased lower extremity position sense in $13(34 \%)$, and decreased vibration sense in 22 $(58 \%)$.

In recruiting the $\mathrm{CO}$ group, all residents $(n=170)$ of a single congregate housing facility were sent a letter requesting volunteers to participate in a study of how older adults rise from the floor. Forty-six responded that they would be interested in participating. Eight could not be scheduled for testing because of acute medical illness (such as a viral syndrome) or because of interference from other ongoing activities, leaving the 38 participants analyzed below.

\section{Equipment}

Subjects rose from a $1.85 \times 1.2-\mathrm{m}$ simulated floor made from $1.9-\mathrm{cm}(3 / 4$-inch) plywood covered with $0.32 \mathrm{~cm}(1 / 8-$ inch) industrial carpeting. Subjects activated a pair of switches to record the rise time for each task: upon initiation of the rise task, a switch was released to begin the time, and upon completion of the rise task a switch was depressed to halt the time.

\section{Protocol}

Subjects were instructed to rise at a comfortable rate from various initial positions on the floor, using any motions they desired. While in their initial body configuration, subjects laid in contact with a switch on the floor surface that activated with the onset of the rise. Subjects pressed a second switch placed on a $0.9-\mathrm{m}$ tripod upon completion of the rise. The subjects rose from the floor to an upright standing position under two conditions: (1) Without support and (2) With support, a $55 \mathrm{~cm} \times 60 \mathrm{~cm} \times 60-\mathrm{cm}$ end table. The support was placed approximately $25 \mathrm{~cm}$ from the subject. For each condition, the subjects assumed the following initial positions: (1) supine (SU), where the subject's head rested on the floor switch, with legs extended and arms extended alongside the body; (2) on side (OS), where the subject lay on the right side of the body with the floor switch under the right shoulder and a pillow to support the neck; (3) prone (PR), where the subject lay in a prone position with the floor switch under the right hand; (4) all fours (AF), where the subject was on hands and knees with shoulders flexed, elbows extended, with the floor switch under the right hand; and (5) sitting (SI), where the subject sat on the floor switch with legs in from of him/her, knees and hips bent, and hands resting on the floor beside each hip. When necessary, $\mathrm{CO}$ subjects were assisted by the experimenter from a standing position to the initial floor rise task position. $\mathrm{CO}$ subjects also wore a 2 -inch wide transfer belt to be used to assist in lowering and as a device to ensure safety. Three trials of each task were performed in the order of the positions given above, two trials without the use of the support (without support) followed by one trial with the use of the support (with support). Only the second without support trial was used for the timing data. One SU and one PR task ("follow-up" tasks) were performed after all of the above tasks to determine any fatigue- or practice-related performance effects. For the final task, subjects were to rise as fast as possible from the SU position ("speed" task).

After subjects completed each task, they compared the difficulty of the task just performed to the difficulty encountered during the SU task. Of all of the tasks performed that might be considered as the reference task for this subjective rise difficulty assessment, the SU task seemed to be the most reasonable because it was unlikely to become an intermediate position for rising from another position (e.g., all fours position might become an intermediate rise position for the starting prone position) and because the SU task has been commonly used in other studies of rising from the floor. ${ }^{9-10}$ Subjects then rated the rise tasks according to a five-point scale: much easier, slightly easier, no difference, slightly harder, and much harder (than SU performance). After completing all of the rise tasks, the subjects selected which one of the rise tasks they thought was the easiest.

\section{Data Analysis}

Total rise time was defined as the time between activation of the floor switch and activation of the tripod switch. Repeated measures analysis of variance was used to test mean total rise time for group, task, and use of support effects. Post hoc testing for task differences within each group was conducted using Scheffe's test for multiple comparisons with a $P$ level set at 0.05 . Questionnaire responses were ranked as 
integers from 1 through 5 , i.e., ranging from 1 for much easier to 5 for much harder. Accordingly, three nonparametric analyses of median questionnaire responses were made: Kruskal-Wallis test to determine group differences according to each task, Friedman test to determine task differences within each group, and Wilcoxon test to determine the effect of support use within each group.

\section{RESULTS}

\section{Task Performance Ability}

Although all of the $\mathrm{YC}$ and $\mathrm{HO}$ were able to successfully complete each task, some $\mathrm{CO}$ were unable to perform a number of the floor rise tasks. Some $\mathrm{CO}$ subjects were unable to complete each task because they: (1) rose successfully but violated the task (e.g., reached onto the experimenter for support); (2) attempted the task but were unsuccessful; (3) refused to attempt the task; or (4) had the task terminated midway through the attempt by the experimenter for fear of potential subject injury (occurring in one subject).

Based on these criteria, many of the $\mathrm{CO}$ were able to complete all (five) or nearly all (four) of the tasks. Fifty-five percent of the $\mathrm{CO}$ completed four to five tasks without support use, and $63 \%$ of the CO completed four to five tasks with support use. On the other hand, some $\mathrm{CO}$ could not rise even with initial position changes and support use (see Table 2a). Twenty-four percent of the $\mathrm{CO}$ (nine subjects) could not complete any of the without support tasks and $13 \%$ of the $\mathrm{CO}$ (five subjects) could not complete any of the with support tasks. Thus, there was a subset of congregate housing residents who were unable to rise from the floor irrespective of the initial position and, to a lesser extent, whether or not a support was used. Note that four of the nine subjects who were unable to rise in without support conditions were able to complete at least one with support condition, leaving the five subjects who could not rise under any condition.

The percent of $\mathrm{CO}$ unable to complete each task ranged from 34 to $45 \%$ for without support and from 21 to $39 \%$ for with support tasks (see Table $2 \mathrm{~b}$ ). The task that $\mathrm{CO}$ were least likely to be unable to perform was on side, with support ( $21 \%$ unable). The task that $\mathrm{CO}$ were most likely to be unable to perform was the prone, without support $145 \%$ unable). Compared to tasks without use of the support, the support was most helpful in assisting $\mathrm{CO}$ to rise from the on side and prone positions, with a small effect in the other positions. Thus, the support helped more $\mathrm{CO}$ rise successfully but only from certain initial positions.

\section{Total Rise Time}

Two analyses of mean total rise time were conducted. The first compared $\mathrm{YC}(\mathrm{n}=24)$ with $\mathrm{HO}(\mathrm{n}=24)$. In the

Table 1. Clinical Characteristics of Young Controls, Community-Dwelling Healthy Older Adults, and Congregate Housing Older Adults

\begin{tabular}{lllr}
\hline \multicolumn{1}{c}{ Characteristic } & Young Controls & Healthy Old & Congregate Old \\
\hline N (female/male) & $24(12 / 12)$ & $24(12 / 12)$ & $38(32 / 6)$ \\
Mean age (range) & & & \\
All & $23(19-30)$ & $73(66-87)$ & $80(63-94)$ \\
Women & & $75(66-87)$ & $81(68-94)$ \\
Mean BMI $\left(\mathrm{kg} / \mathrm{m}^{2}\right)$ & 22 & 23 & 26 \\
All & & 23 & 28 \\
Women & & 29 & \\
Mean Folstein Mini-Mental & & \\
State score & & \\
\hline
\end{tabular}

Percent of subjects with abnormal finding on medical history and physical examination

History/Examination

Healthy Old (\%) Congregate Old (\%)

$\begin{array}{lrr}\text { History } & & \\ \text { Rare back/leg pain } & 17 & 21 \\ \text { Daily back/leg pain } & 0 & 34 \\ \text { Falls in past year } & 8 & 26 \\ \text { Poor balance } & 0 & 47 \\ \text { Requires assistive device for ambulation } & 0 & 21 \\ \text { Examination } & & 92 \\ \text { Altered lower extremity reflexes } & 42 & 58 \\ \text { Decreased vibration sense } & 13 & 34 \\ \text { Decreased position sense } & 0 & 26 \\ \text { Upper extremity weakness } & 0 & 37 \\ \text { Lower extremity weakness } & 0 & 24 \\ \text { Positive Romberg test } & 0 & 0\end{array}$


Table 2. Percent of Congregate Housing Group Unable to Complete Rise by Initial Position and Number of Rise Tasks

\begin{tabular}{llllll}
\hline & \multicolumn{5}{c}{ a. Initial Position } \\
\cline { 2 - 6 } & Supine & On side & Prone & All fours & Sitting \\
\hline Without support & $34(\%)$ & $39(\%)$ & $45(\%)$ & $39(\%)$ & $39(\%)$ \\
With support & 32 & 21 & 29 & 39 & 34 \\
\hline
\end{tabular}

\begin{tabular}{lllcccc}
\hline & \multicolumn{5}{c}{ b. No. of Tasks Unable to Perform } \\
\cline { 2 - 6 } & 0 & 1 & 2 & 3 & 4 & 5 \\
\hline Without support & $39(\%)$ & $16(\%)$ & $8(\%)$ & $8(\%)$ & $5(\%)$ & $24(\%)$ \\
With support & 39 & 24 & 11 & 5 & 8 & 13 \\
\hline
\end{tabular}

second comparison, because some of the CO subjects were unable to complete certain rise tasks and because of the small number of $\mathrm{CO}$ males available to be tested, we compared $\mathrm{HO}$ women (group HOF, $\mathrm{n}=12$ ) with CO women who were able to complete at least one of the tasks (group COF, $n=27$ ).

\section{Comparison of Groups}

YC and $\mathrm{HO}$ mean total rise time for each without support task appears in Figure 1, whereas HOF and COF without support rise time data appear in Figure 2. In general, HO take twice as long to rise than do YC for most of the tasks, with $\mathrm{HO}$ ranging from 2.4 to 5.5 seconds and $\mathrm{YC}$ ranging from 1.4 to 2.6 seconds (group effect $P<.0001$ ). By comparison, $\mathrm{COF}$ take two to three times longer to rise than HOF, with COF ranging from 6.4 to 13.2 seconds and HOF ranging from 3.1 to 6.4 seconds (group effect $P<.0001$ ).

\section{Comparison of Support Use}

Surprisingly, there was no reduction in rise time with support use. Mean rise time in each group actually tended to rise with support use (range in YC 1.6-3.1, in HO 2.8-5.7, in HOF 3.2-6.7, and in CO 11.0-15.5 seconds), as compared to no support use, although these increases tended to be statistically insignificant.

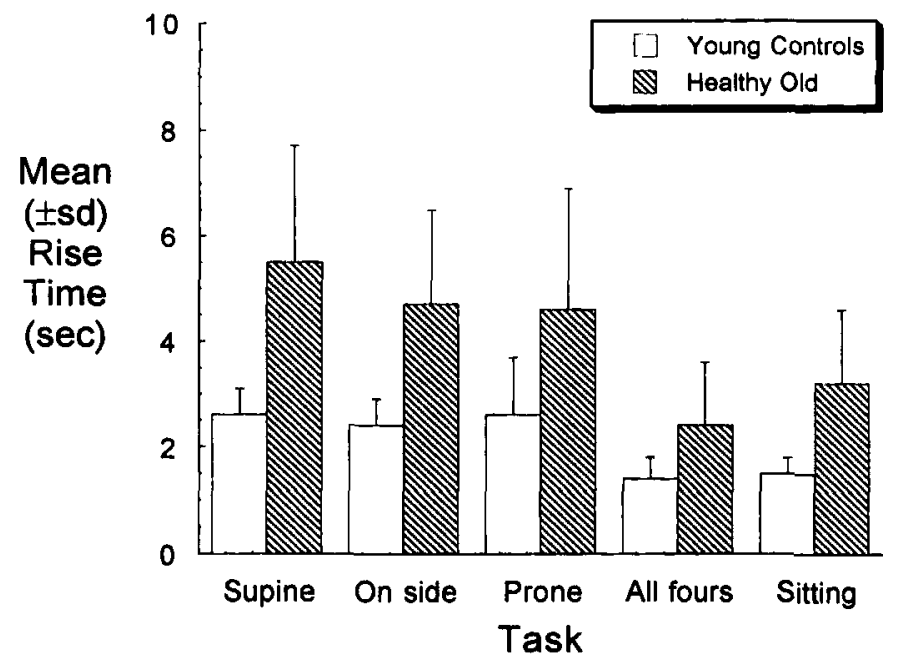

Figure 1. Mean rise time (seconds) in young control subjects $(\mathbf{n}=$ $24)$ and healthy old $(n=24)$ during without support floor rise tasks. Error bars represent standard deviations.

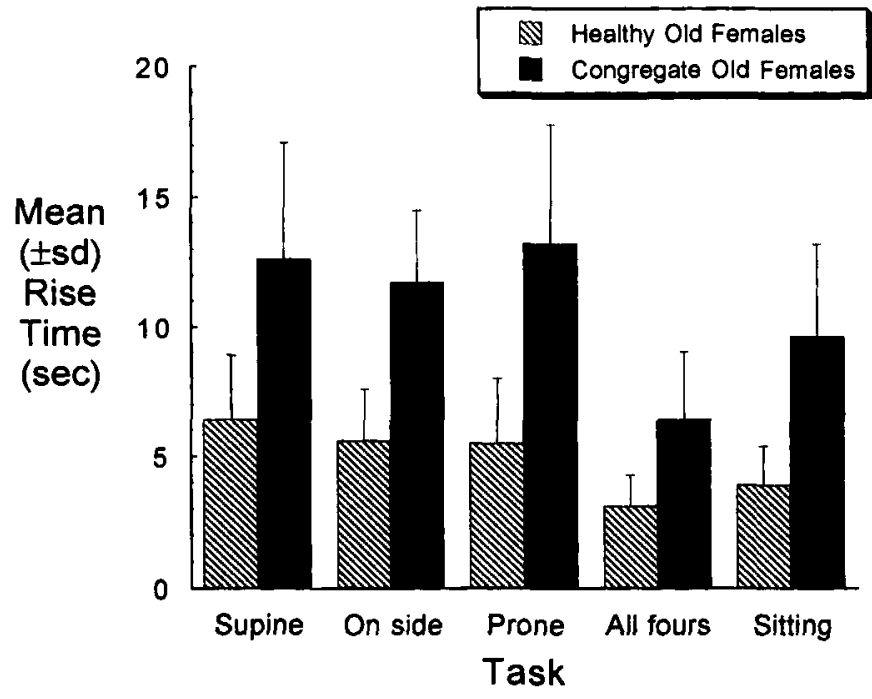

Figure 2. Mean rise time (seconds) in healthy old female subjects $(n=12)$ and congregate old female subjects (total $n=27$ ) during without support floor rise tasks. Some of the congregate old could not do every task; thus the $n$ for each task differs for without support ( $\mathrm{SU}, \mathrm{n}=21 ; \mathrm{OS}, \mathrm{n}=19 ; \mathrm{PR}, \mathrm{n}=17 ; \mathrm{AF}, \mathrm{n}=$ 19 ; SI, $n=18$ ), and with support ( $\mathrm{SU}, \mathrm{n}=24 ; \mathrm{OS}, \mathrm{n}=26$; $\mathrm{PR}$, $\mathrm{n}=23 ; \mathrm{AF}, \mathrm{n}=19 ; \mathrm{SI}, \mathrm{n}=20)$. Error bars represent standard deviations.

\section{Comparisons of Tasks}

Overall, rising from all fours (AF) was performed the most quickly by all groups under both support conditions (overall task effect $P<.0001$ ). Group mean rise time was reduced by up to $50 \%$, for example, when comparing AF to SU (supine position). Smaller reductions in rise time were seen in COF when comparing $\mathrm{AF}$ to the other positions under support use conditions. Subjects also generally rose more quickly from the sitting position (SI) than the supine (SU), prone (PR), or on side positions (OS), mostly among YC and HO ( $P$ usually $<0.005$ using Scheffe comparisons within both support conditions). Generally, no significant rise time differences were seen among SU, PR, and OS in any of the groups.

\section{Follow-up and Speed Tasks}

Subjects generally performed the follow-up supine and prone tasks slightly faster than the previous tasks, with a mean change of 1 second or less, i.e., a 1 to $12 \%$ improve- 
ment $(P<.005$ overall). Thus, there is no evidence of a fatigue effect throughout testing, and the practice effect is small. All groups rose faster during the supine "speed" task, with mean improvements of $34 \%$ in $\mathrm{YC}, 29 \%$ in $\mathrm{HO}$, and $22 \%$ in COF $(P<.001$ overall $)$.

\section{Task Difficulty Rating}

Upon comparing the difficulty encountered during each task with the difficulty experienced during the SU task (see Fig. 3), median without support and with support ratings ranged from 1.0 to 4.0 in $\mathrm{YC}, 2.0$ to 3.0 in $\mathrm{HO}$, and 1.5 to 3.0 in COF groups. The AF task was rated as easier to perform based on median group difficulty rankings (by Friedman test: YC without and with support, scores of 1.0 and 2.0, respectively, $P<.0001$; HO without support, score of $2.0, P<$ .001 ; and $\mathrm{CO}$ with support, score of $1.5, P<.05)$. All three groups tended to rank the tasks similarly with the exception of SI with support; YC (median 2.0) rated the task easier than $\mathrm{HO}$ (median 2.5), whereas $\mathrm{HO}$ rated the task easier than $\mathrm{CO}$ (median 3.0) $(P<.05$ by Kruskal-Wallis test). Overall, the use of the support did not strikingly reduce the perceived task difficulty, even in the $\mathrm{CO}$ as might be expected. These questionnaire findings were also true for $\mathrm{COF}$ as well, where the AF task was rated as the easiest to perform (by Friedman: $P<$ .02 , with support) and where use of a support did not reduce task difficulty.

\section{DISCUSSION}

Older adults have more difficulty rising from the floor than young adults. The healthy old took twice as long as the young controls to rise, and the congregate old took two to three times as long as the healthy old. There is also a subset of congregate housing residents who were unable to rise successfully. Healthy old-congregate old differences in floor rise performance appear linked to the degree of physical impairment in the congregate old. The congregate old tended to have more pain, weakness, and/or balance impairments. Mechanisms contributing to the young-healthy old differences are more subtle. Previous studies of rising from a supine to a sitting position at the edge of the bed suggest that the healthy old, as compared to young adults, use more trunk rotation, more lateral trunk flexion, and more elbow support, ${ }^{15,16}$ i.e., a suggestion that age-related decrements in trunk function may alter bed rise strategies. Further studies are needed to determine how floor rise strategies may differ between healthy young, healthy old, and more physically impaired old adults.

The most favorable initial position and support use conditions depend to some extent on the group and outcome analyzed. Congregate housing subjects overall were most likely to be able to rise when starting from a side-lying position using the table support. Other congregate housing subjects who were able to successfully rise from at least one position (COF), as well as healthy old and young control subjects, rose more quickly and admitted to least difficulty when rising from an all fours position. Given that the trunk was already elevated off the floor, one might expect that the all fours position would yield the fastest rise time and be rated as the easiest to perform. Yet, in terms of facilitating rise success in a frail population, the all fours position may not be the most preferred. Other positions and rise conditions had less of an effect. Healthy old and young control subjects rose more quickly from an initial sitting position (again, not surprising given that the trunk is already elevated), but the effect was not as strong as that seen with the all fours position. Based on the timing and questionnaire data, there are no apparent advantages to starting from a supine, prone, or on side position. Future studies should explore the biomechanical requirements of rising from these initial positions and determine whether getting into and rising from an all fours position is biomechanically favorable for frail older adults.

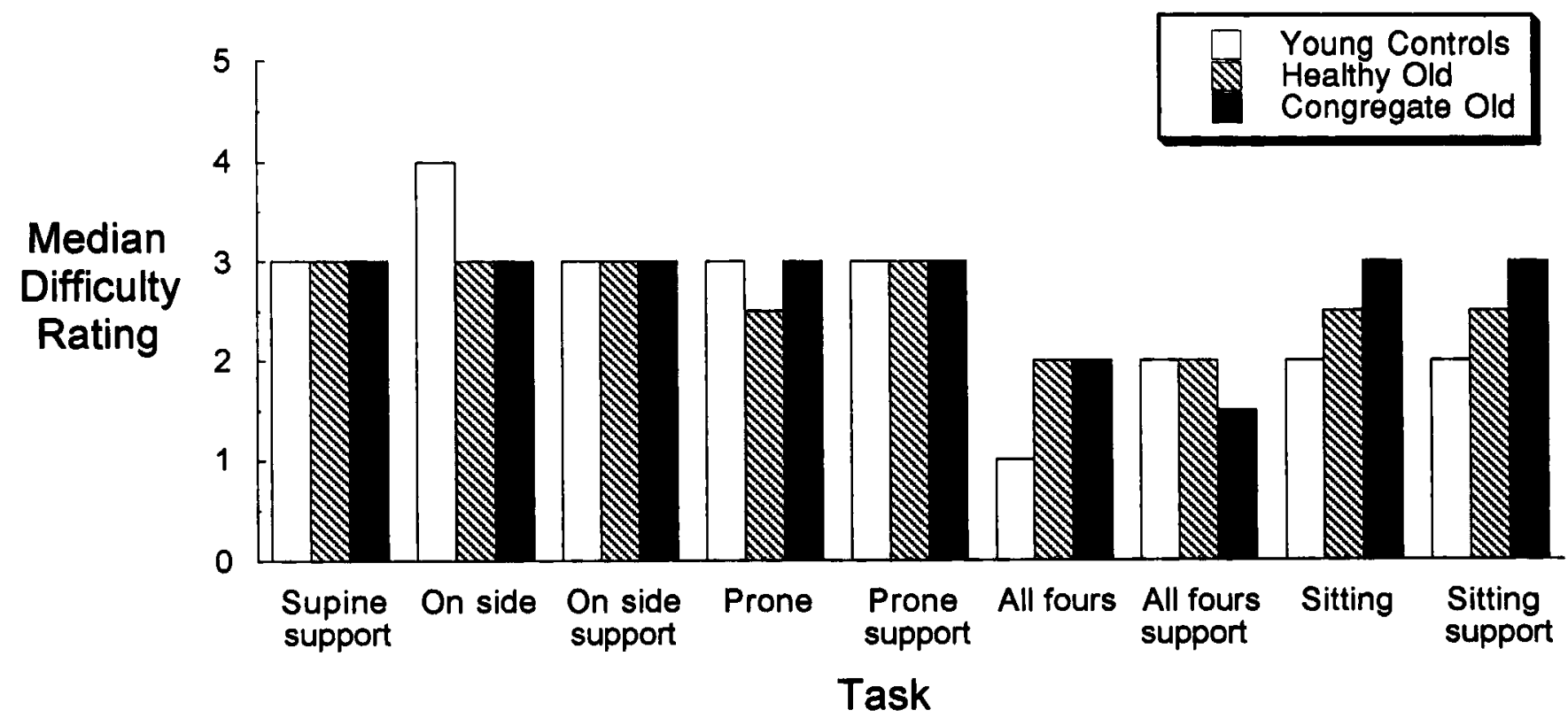

Figure 3. Median difficulty ratings by young control subjects, healthy old, and congregate old in without and with support tasks. Ratings represent the perceived difficulty for each task as compared with the difficulty encountered while rising from SU (supine) without support. Lower numbers represent less perceived difficulty, i.e., a score of 1 for much easier and 5 for much harder. 
Surprisingly, there were no significant advantages to using a support to assist with rising among those who were able to successfully complete the rise (specifically the young controls and healthy old). However, among the congregate old, four subjects who could not complete a without support task became able to complete a with support task. Furthermore, the congregate old were most able to rise from the on side position while using the support. Perhaps the support is most accessible from an on side position and thus becomes a more critical contributor to rise success. Yet, there still remained five subjects who could not rise even when the support was provided. Perhaps there are some frail older adults who might be able to benefit from support use and others who might not. Further research is needed to explore the interaction between physical impairment, initial body configuration, and accessibility of (and design of) devices to best facilitate rising from the floor.

Data from this study may also serve as the foundation for future interventions to improve the ability to rise from the floor. The optimal approach to teaching floor rise skills has yet to be identified and even if such a program existed, there is evidence that patients might avoid such training due to the fear evoked by being placed on the floor, i.e., in a situation from which they could not rise. ${ }^{8}$ Pilot data suggest that floor rise training can be successful in Parkinson's disease patients. ${ }^{14} \mathrm{~A}$ better understanding of the various rise strategies, including the use of furniture/assistive devices in an appropriate manner, would be useful in developing future floor rise training programs.

\section{ACKNOWLEDGMENTS}

The authors gratefully acknowledge the assistance of Linda Nyquist and the Human Subjects Core of the Claude Pepper Older Adults Independence Center, and Rhonda Keller, Julie Grunawalt, Ann Van Wagoner, Jodi Buck, Jenny
Myers, Cynthia Lockman, and the staff and residents of Lurie Terrace.

\section{REFERENCES}

1. Tinetti ME, Liu WL, Claus EB. Predictors and prognosis of inability to get up after falls among elderly persons. JAMA 1993;269:65-70.

2. Campbell AJ, Borrie MJ, Spears GF et al. Circumstances and consequences of falls experienced by a community population 70 years and over during a prospective study. Age Ageing 1990;19:136-141.

3. Nevitt MC, Cummings SR, Hudes ES. Risk factors for injurious falls: A prospective study. J Gerontol 1991;46:M164-M170.

4. Vellas B, Cayla F, Bocquet $\mathrm{H}$ et al. Prospective study of restriction of activity in older people after falls. Age Ageing 1987;16:189-193.

5. Wild D, Nayak USL, Isaacs B. How dangerous arc falls in older people at home? BMJ 1981;282:266-268.

6. Mallinson WJW, Green MF. Covert muscle injury in aged patients admitted to hospital following falls. Age Ageing 1985;14:174-178.

7. Tinetti, ME, Richman D, Powell L. Falls efficacy as a measure of fear of falling. J Gerontol 1990;45:P239-P243.

8. Simpson JM, Salkin S. Are elderly people at risk of falling taught how to get up again? Age Ageing 1993;22:294-296.

9. Van Sant AF. Rising from a supine to erect stance: description of adult movement and a developmental hypothesis. Phys Ther 1988;68:185-192.

10. Van Sant AF. Age differences in movement patterns used by children to rise from a supine to erect stance. Phys Ther 1988;68:1330-1339.

11. Unrau K, Hanrahan SM, Pitetti KH. An exploratory study of righting reactions from a supine to standing position in adults with Down syndrome. Phys Ther 1994;74:1116-1124.

12. Ford-Smith CD, Van Sant AF. Age differences in movement patterns used to rise from a bed in subjects in the third through fifth decades of age. Phys Ther 1993; $73: 300-309$.

13. Green $\mathrm{L}$, Williams K. Differences in developmental movement patterns used by active versus sedentary middle-aged adults coming from a supine position to erect stance. Phys Ther 1992;72:560-568.

14. Yekutiel MP, Pinhasov A, Shahar G et al. A clinical trial of the re-education of movement in patients with Parkinson's disease. Clin Rehabil 1991;5:207214.

15. Alexander NB, Fry-Welch D, Ward M et al. Quantitative assessment of bed rise difficulty in young and older women. J Am Geriatr Soc 1992;40:685691.

16. Alexander NB, Fry-Welch D, Marshall LM et al. Healthy young and old women differ in their trunk elevation and hip pivot motions when rising from supine to sitting. J Am Geriatr Soc 1995;43:338-343. 